\title{
A control-oriented dynamic model of discrete manufacturing systems
}

\author{
E. Canuto \\ Dipartimento di Automatica e Informatica, Politecnico di Torino, \\ Corso Duca degli Abruzzi 24, I 10129 Torino, Italy \\ e-mail: canuto@polito.it-fax: +3911654 7099
}

\begin{abstract}
The paper presents a mathematical model of discrete-manufacturing production systems in terms of discrete-time state equations; the model allows to analyse their dynamics under autonomous evolution and real-time feedback control.
\end{abstract}

\section{Keywords}

Discrete manufacturing systems, factory dynamics, production systems.

\section{INTRODUCTION}

In factories where discrete manufacturing takes place, objects of various types circulate and some of them are progressively transformed and assembled into commercial finishedproducts: raw materials, components, semifinished and finished products, fixtures, equipments, tools. The ensemble of such objects can be mathematically described (Canuto et alii, 1993) by a countable and finite set of (manufacturing) objects. Based on this concept, a (manufacturing) algebra has been introduced, capable of describing and manipulating, in a formal way and independently of any technological sector, the set of manufacturing operations to be carried out on such objects. Its key elements are:

- A generic definition of manufacturing operations, capable of encompassing any type of operation typical of discrete manufacturing processes, like assembling, disassembling, transporting, machining, setting-up, fixturing.

- Composition rules to aggregate elementary operations and to describe in a formal and compact way any complex network of operations designed to be fed by raw materials and to yield a set of finished-products.

The key elements of the algebra, i.e. objects and manufacturing operations, have been subsequently used to lay down the foundations of a dynamic model of the factory production processes. The basic concept, very often forgotten but of paramount importance in control theory, is the distinction between a factory plant and its production control system. It is the lat- 
ter that makes the factory alive, launches and controls production processes, determines the inflow and the inflow-places of raw materials, dispatches transport operations, commands workstations and their manufacturing operations, decides drawing of finished products. The models developed in the present paper mainly concern the factory plant. Production control systems are barely mentioned, being introduced as subsystems capable of modifying the factory dynamics.

\section{DEFINITIONS}

Let us start with some definitions.

Manufacturing process. It is the output of the engineering work, defining the objects and the manufacturing operations needed to manufacture the desired finished-products. Manufacturing processes are not bounded to the factory where they will be performed. They are described with an algebra, capable of operating on the object set through algebraic operations transforming a set of objects into another set.

Factory. A factory is defined as a set of:

- manufacturing units: machine tools, presses, robots, or simply working stations where workers can perform specific operations;

- transport units;

- storage units: every factory space where objects can be placed, also temporarily.

Production system. It is defined as a pair: a factory with a production plan together with a production control system capable of ensuring the plan achievement. The factory dynamics describes the production systems, i.e. factory equipped with production plan and control.

Let us remark that production controls are often achieved though automatic devices and consequently the are embedded in the factory plant itself. It is however conceptually important to distinguish between the plant to be controlled and the control itself. To give a simple example, think to storage units that are managed either through a FIFO rule, first-in-first-out, either through a LIFO rule, last-in-first-out, or with random supply and drawing. The first two cases are storage units equipped with a control rule and possibly an automatic control device performing the rule; the rule and the relevant device must be retained separated from the storage unit.

\section{THE ALGEBRA OF MANUFACTURING PROCESSES}

\subsection{The manufacturing objects}

\section{Types of objects}

It is assumed that all the different object types circulating in the factory and entering the production process be elements of a countable and finite set $\boldsymbol{O}$, having cardinality $n_{k}$. In this way the set $\boldsymbol{O}$ can be ordered as a list and each object type is univocally defined by the index $k$. A 
basic assumption is that such a list, although very large (also thousands of hundreds of objects) be available.

\section{Quantities of objects}

Production processes in discrete-type manufacturing deal with quantities of objects: the stock level of a store, the input lot size to a manufacturing operation, the yield of a manufacturing operation, quantities of objects transported from one place to another. Quantities of objects are denoted by a vector $q$ of size $n_{k}$, whose generic entry $q(k)$ is an integer number (positive or negative) denoting the available or missing quantity of the $k$ th object type. The set of the quantity vectors is denoted by $Q$ and it can be treated as an integer vector space.

\subsection{The manufacturing operations}

\section{Definition}

A generic manufacturing operation $A$ has been defined to be the pair $A=(\boldsymbol{u}, \boldsymbol{y})$ of an input quantity vector $\boldsymbol{u}$ (components) and an output quantity vector $\boldsymbol{y}$ (products). A set of manufacturing operations is denoted by $\boldsymbol{A}$. To each operation $A$ specific functionals can be associated like the manufacturing time $\tau(A)$.

\section{Product map}

The concepts of object quantity and manufacturing operation, together with the algebra introduced in (Canuto et alii, 1993), allow to define for each list of finished-products a triple $\boldsymbol{M}=\{\boldsymbol{O}, \boldsymbol{Q}, \boldsymbol{A}\}$, called product map, where $\boldsymbol{O}$ is the set of the objects needed for their production, $\boldsymbol{Q}$ is the quantity space of the objects and $\boldsymbol{A}$ is a finite set of the elementary manufacturing operations, needed to produce the list of finished-products. A generic operation $A$ of $\boldsymbol{A}$ will be denoted also with $A_{s}, s$ being the index of the operation in $A, s=1, \ldots, n_{s}$.

Using algebraic compositions (parallel and series) it is possible to create aggregate operations over the set $\boldsymbol{A}$. Given a subset $S\left\{A_{1}, \ldots, A_{h}, \ldots, A_{n}\right\} \subset \boldsymbol{A}$, an aggregate operation over $S$ is defined as an arbitrary composition of the such operations and it will be denoted by $W=c\left(A_{1}, \ldots, A_{h}, \ldots, A_{n}\right)=(\boldsymbol{u}(W), y(W))$, where $\boldsymbol{u}(W)$ and $\boldsymbol{y}(W)$ are the input and output vectors of the aggregate operation. To each aggregate operation a quantity vector $\boldsymbol{m}(W)$ is also associated, whose generic element $m(h)$ equals the repetition number of the operation $A_{h}$ in the aggregate operation $W$.

Using graphical symbols, any product map can be given a graphical representation, showing operations and object interconnections as well as object quantities. The manufacturing operations will be represented by rectangles; the object types by circles, the input and output vectors of a manufacturing operation by arrows connecting circles to rectangles and vice versa; each arrow is drawn with the object quantity aside.

\section{A simple example}

A simple example of product map with two finished-products is sketched in Figure 1; the following manufacturing times are assumed: $\tau\left(A_{1}\right)=4, \tau\left(A_{2}\right)=5, \tau\left(A_{3}\right)=4, \tau\left(A_{4}\right)=6, \tau\left(A_{5}\right)=6$, $\tau\left(A_{6}\right)=8, \tau\left(A_{7}\right)=5, \tau\left(A_{8}\right)=7$. 


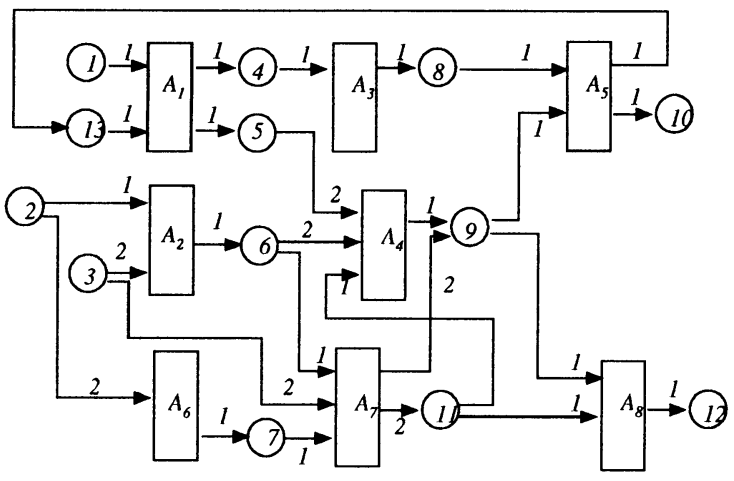

Legenda

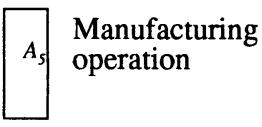

(11) Object type

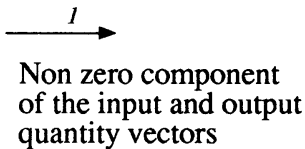

Figure 1 A simple product map.

\section{Object classification}

Given a product map, it is possible to classify objects into four classes according to definitions given in (Canuto et alii, 1994).

- Raw materials: no elementary operation of the map includes them as input objects; in the above example they are indexed by $k=1,2,3$.

- Semifinished objects: they are both employed or better consumed (input objects) and produced (output objects) by elementary operations; in the above example they are indexed by $k=4,5,6,7,8,9,11$.

- Reusable materials: they are objects which are employed without being consumed by elementary operations and consequently they may re-circulate; in the above example they are indexed by $k=13$.

- Finished-products: they are objects which are only produced by elementary operations and do not appear as input objects; in the above example they are indexed by $k=10,12$.

\section{Manufacturing plan.}

A specific composition $W(\boldsymbol{A})$ made from the whole set of elementary operations of $\boldsymbol{A}$ can be defined as a manufacturing plan: it yields as product the output quantity vector $y(W)$, it has a Bill of Materials equal to the input quantity vector $\boldsymbol{u}(W)$ and a manufacturing time $\tau(W)$. In case of more than one finished-products the vector quantity $\boldsymbol{y}(W)$ defines the product mix. Moreover the repetition vector $\boldsymbol{m}(W)$ defines how many times each operation has to be repeated. Thus a manufacturing plan can be defined as a quintuple $\{W(\boldsymbol{A}), \boldsymbol{u}(W), \boldsymbol{y}(W), \boldsymbol{n}(W), \tau(W)\}$.

Specifically, given an integer product mix $\boldsymbol{p}$, the aggregate operation $W(\boldsymbol{A})$ having as output vector the product mix, i.e. such that $\boldsymbol{y}(W)=\boldsymbol{p}$, is in general not unique. If $W(\boldsymbol{A})$ exists, it can range from compositions reducing the working time (when operations are mostly composed in parallel) to compositions reducing norms of the input quantity vector $u(W)$ (when operations are mostly composed in series). 
Using the previous example, given the mix: $p(10)=4 x$ and $p(12)=2 y, x$ and $y$ being integers $\geq 0$, the aggregate operation:

$W=\left(\alpha A_{5}+\eta A_{8}\right)\left(\delta A_{4}\right)\left(\alpha A_{3}+\gamma A_{7}\right)\left(\alpha A_{1}+\beta A_{2}+\gamma A_{6}\right)$

can be shown to possess the minimum-norm Bill of Materials, i.e. $u(W)$ includes only raw objects and fixtures, i.e. $u(l)=4 x, u(2)=2 y+6 x, u(3)=4 y+12 x, u(13)=4 x, u(i)=0,4 \leq k<13$. Moreover, within the the whole set of aggregate operations having a minimum-norm Bill of Materials, $W$ can be shown to possess the least manufacturing time $\tau(w)$ :

$\tau(W)=\max \left\{\tau\left(A_{5}\right), \tau\left(A_{8}\right)\right\}+\tau\left(A_{4}\right)+\max \left\{\tau\left(A_{3}\right), \tau\left(A_{7}\right)\right\}+\max \left\{\tau\left(A_{1}\right), \tau\left(A_{2}\right), \tau\left(A_{6}\right)\right\}=26$

Finally, the repetition vector $\boldsymbol{m}(W)$ yielding the product mix $\boldsymbol{p}$ holds:

$m\left(A_{1}\right)=m\left(A_{3}\right)=m\left(A_{5}\right)=\alpha=4 x, m\left(A_{2}\right)=\beta=5 x+y, \quad m\left(A_{6}\right)=m\left(A_{7}\right)=\gamma=x+y, m\left(A_{4}\right)=\delta=2 x$, $m\left(A_{8}\right)=\eta=2 y$.

\section{THE ELEMENTS OF THE FACTORY DYNAMICS}

In (Canuto et alii, 1993) it has been argued that a factory can be modelled as a network made of three different mathematical elements:

- storage units,

- transport units,

- manufacturing units.

Storage units correspond to space volumes in the factory building, where objects can be temporarily laid up for any convenience.

Transport units connect storage units and, whenever required by the production control system, transfer objects from one place to another. Transport operations are specified by the type and quantity of the objects to be moved from the origin to the destination places.

Manufacturing units draw objects to be manufactured, from a specific storage unit (the input store), and lay up their manufactured product in the output store. Each unit can carry out a set of admissible operations: the operations to be performed are selected and commanded by the production control system.

The factory model is assumed to evolve only at discrete times; in other words the evolution of the production process is sampled only at time instants separated by a finite time interval $T$, called sampling step. Therefore the factory mathematical model will be of the following type:

$x(t+1)=F[x(t), u(t)], \quad x\left(t_{0}\right)=x_{0}$

being $\boldsymbol{x}$ the state vector, $\boldsymbol{u}$ the input vector, $\boldsymbol{x}_{0}$ the initial state. Only the state and the input vectors are assumed to vary in time; the factory layout is assumed to be time-invariant. A generic time interval $[\tau, \tau+T)$ during which the $t$-th sample of the factory state is obtained will be indicated as the $t$-th time step.

Factory modelling, still being always an approximate description, can be made at various 
levels of detail depending upon the scope. Here, since the main goal is a dynamic analysis, the frequency band of interest has to be defined. In a production process, different transient phenomena can be observed: fast transients related to start-stop of manufacturing and transport units, slow transients due to fluctuations of object quantities in storage units and in the whole factory.

A common treatment of both fast and slow dynamics would make the model too involved, without a specific advantage. When focusing on fast dynamics, usually it is not necessary to look at the whole factory. Instead it is preferable to analyze the single manufacturing units, modelling transitions between different tooling equipments or setups or, for a specific setup, transitions between operative and wait states. When the interest is on slow dynamics, i.e. on drifts and trends of materials and semifinished products in storage units, one should model the whole factory, but could also greatly simplify the model of manufacturing and transport units, by neglecting their fast transients.

In both cases, it is of paramount importance to choose the right time unit or sampling step $T$. A rule of thumb is to make it sufficiently shorter than the time constants of the transient phenomena to be described, but longer than the transients to be neglected. Hence, to investigate slow factory dynamics, the sampling step has to be longer than manufacturing and transport times and such that, during any single step, the units can carry out several operations without accounting for starts and stops of the single operations.

\subsection{The storage units}

The basic assumption is that in the factory a finite list $S$ of places where to store objects be available. Each place will be a storage unit, indicated by the index $i, i \leq n_{i}$, where $n_{i}$ is the cardinality of $\boldsymbol{S}$. Storage units are dynamic elements modelled by adders. The list of the object quantities, or stock level, available during the $t$-th time step, is the state vector of the unit and is indicated by the $n_{k}$-sized vector $\boldsymbol{x}(i, t)$. The current value of $\boldsymbol{x}(i, t)$ is the results of a sequence of storage and drawing operations occurred in the past time steps $\tau<t$. To each storage unit a very small subset of objects is usually assigned: such an assignment problem must be considered as a planning problem which is here retained solved.

To model the whole factory, the state vectors of the single storage units are composed into a bi-dimensional vector $x(t)$, sized $n_{i} \times n_{k}$, whose generic component will be $x(i, k, t), i$ being the storage unit index and $k$ the object type. In a similar way, any list of object quantities, which is referred to the whole set of the storage units - like drawn or stored quantities will be denoted by bi-dimensional vectors $\mathbf{y}$.

The state equations describing the evolution at discrete times $t$ of the whole factory stock level will be:

$x(t+1)=x(t)+y_{S}(t)-y_{D}(t), \quad x\left(t_{0}\right)=x_{0}$

having denoted with $y_{S}(t)$ and $y_{D}(t)$ the amount of objects respectively supplied to and drawn from all the storage units during the $t$-th time step.

The fact that the state vectors of the storage units are highly sparse should not be a worry; reduction of sparsity becomes an issue only when software is designed and implemented; thus it shall be solved with appropriate design methods. 


\subsection{The manufacturing units}

The basic assumption is that the set of manufacturing units $\boldsymbol{W}$ be finite; the generic unit will be denoted with the index $r$ and their total number with $n_{r}$. Each manufacturing unit can perform a very small subset of the elementary operations belonging to $A$; the problem of assigning such subset of operations to each unit is still a planning problem which is retained here solved. We remark that the operation set $\boldsymbol{A}$ is finite, the operation index is denoted with $s$ and the total number of operations with $n_{s}$.

Manufacturing units operate upon commands of the production control system; in other words, as soon as the command of performing the operation type $A_{s}=\left(\boldsymbol{u}_{s}, \boldsymbol{y}_{s}\right)$ has been received, the unit will draw at predefined time instants the input objects included in $\boldsymbol{u}_{s}$ and it will supply, always at predefined time instants, the output objects included in $\boldsymbol{y}_{\boldsymbol{s}}$. Drawing and supply will be made from/to predefined storage units.

If fast dynamics has to be described, a sampling step shall be selected which is less than the time intervals between the command reception and the drawing and supply times; in this case a manufacturing unit behaves like a set of delay lines. Instead, if only slow dynamics has to be described, drawing and supply times are assumed to be indistinguishable and happening during the same sampling step; their net effect on the input and output stores will become apparent only at the next step. Under such assumption, manufacturing units are no more dynamic elements and the factory state reduces to stock level, i.e. the object quantities in the storage units during a sampling step $t$.

In case of slow dynamics, the command vector $\boldsymbol{u}(t)$ is a bi-dimensional vector, whose generic component $u(r, s ; t)$ equals the number of operations of type $s$ have been commanded to unit $r$ at step $t$.

The net effect of the command vector on the stock level $x(t)$ is given by the matrix product $M u(t)=\left(M_{S}-M_{D}\right) u(t)$, where:

- $M_{S}$ is a multidimensional matrix whose generic element $w_{D}(i, k ; r, s)$ equals the quantity of the objects of type $k$ supplied to storage unit $i$ during the performance of the manufacturing operation $s$ on the unit $r$.

- $M_{D}$ is similar to $M_{S}$ and its elements equal the object quantities drawn from the different storage units during the performance of the different manufacturing operations.

The matrix $M$ and the vector $\boldsymbol{u}$ are called, respectively, manufacturing matrix and manufacturing control vector. It should be clear that the previous description assumes that each manufacturing unit possesses a production plan and a control system capable of fulfilling commands during the sampling step $T$; any deviation from such an assumption has to be retained a modelling error.

\subsection{The transport units}

The construction of a generic model, valid for all the transport units, is a very complex task since transport techniques can vary widely from factory to factory. Different transport systems require different models, but material handling and transport are usually regarded as quite independent problems, to be solved by designing specific transport subsystems capable of ensuring that part handling will be free of queues which might deteriorate production 
plans. But if any transport system is considered to possess its own control system and hence is able to perform the commanded operations in well defined time intervals, the same considerations made for manufacturing units apply. The only difference is that the transport operations are a particular type of the manufacturing operations defined by the algebra; they are identity operations since $\boldsymbol{u}_{l}=\boldsymbol{y}_{l}$, i.e. the input and output quantity vectors of a transport operation $A_{l}$ are equal.

The set of transport units is assumed to be finite and denoted with $\boldsymbol{H}$; a generic unit will be indicated by $h, h=1, \ldots, n_{h}$. Each transport unit is able to perform a limited number of transport operations from store to store, being commanded by the factory production control system. Also in this case fast and slow dynamic models are possible; in case of slow dynamics transport units are static elements, since drawing and supply of single operation are assumed to be concentrated during a single sampling step.

The command vector $v(t)$ is a bi-dimensional vector having $v(h, l ; t)$ as a generic element. The effect of a set of commanded transport operations during a step $T$ on the factory stock level is modelled by the matrix product $H v(t)$. The matrix $H$ and the vector $v$ are called, respectively, transport matrix and transport control vector.

\subsection{A simple example of factory network}

In Figure 2 a simple factory network for implementing the product map of Figure 1 is illustrated. Each manufacturing unit is represented by a numbered square, $r=1, \ldots, 10$, with the assigned operations on one side. Each storage unit is indicated by a numbered circle, $i=1,2,3,4$. Each transport unit by a double square, $h=1,2,3$, having the assigned operation on one side.

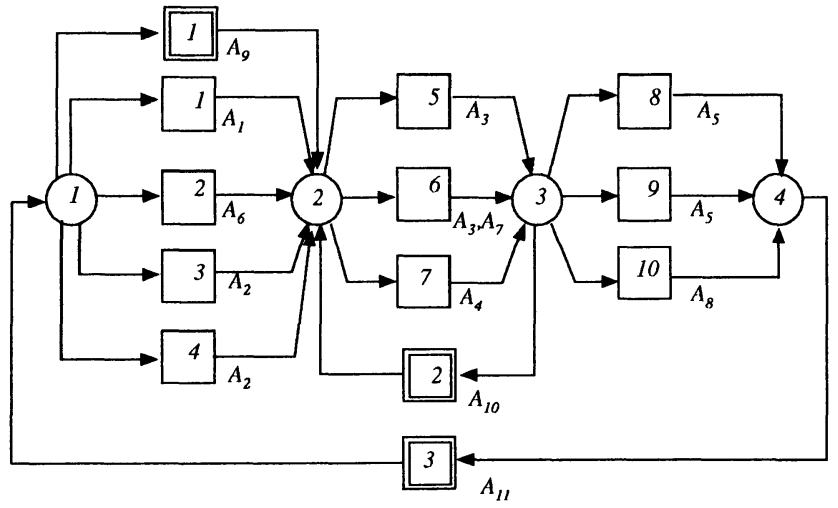

Legenda

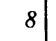

Manufacturing unit

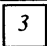

Transport unit<smiles></smiles>

Storage unit

Figure 2 A simple factory network

Let us note that storage units have to be considered without any supplying or drawing rule, i.e. random access; thus any assigned object can be supplied or drawn at any time step in the needed quantity. The elements of the factory and the relevant objects and operations are reported in Table 1. 
Table 1 The factory elements.

\begin{tabular}{|c|c|c|c|c|c|c|}
\hline \multicolumn{2}{|c|}{ Storage units } & \multicolumn{2}{|c|}{ Manufacturing units } & \multicolumn{3}{|c|}{ Transport units } \\
\hline Index $i$ & $\begin{array}{l}\text { Assigned } \\
\text { objects } k\end{array}$ & Index $r$ & $\begin{array}{l}\text { Admissible } \\
\text { operations s }\end{array}$ & Index $h$ & $\begin{array}{l}\text { Admissible } \\
\text { operations l }\end{array}$ & Object $k$ \\
\hline 1 & $1,2,3,13$ & 1 & 1 & 1 & 9 & 3 \\
\hline 2 & $3,4,5,6,7,11$ & 2 & 6 & 2 & 10 & 11 \\
\hline 3 & $8,9,11$ & 3 & 2 & 3 & 11 & 13 \\
\hline \multirow[t]{7}{*}{4} & $10,12,13$ & 4 & 2 & & & \\
\hline & & 5 & 3 & & & \\
\hline & & 6 & 3,7 & & & \\
\hline & & 7 & 4 & & & \\
\hline & & 8 & 5 & & & \\
\hline & & 9 & 5 & & & \\
\hline & & 10 & 8 & & & \\
\hline
\end{tabular}

\section{THE EQUATIONS OF THE SLOW DYNAMICS}

\subsection{The state equations}

\section{The equations}

Since only the slow phenomena of factory dynamics will be accounted for, a sampling step $T$ sufficiently long with respect to manufacturing and transport times is assumed. At time step $t$, the state $x(t)$ measures the stock level of the whole factory. It is assumed that the transport and manufacturing control vectors $u(t)$ and $v(t)$, to be actuated during the time step, have been computed by the control system taking into account the current factory plant capacity, hence ensuring that all the operations will be completed during the same step. The state of the storage units at the next time step $t+1$ holds:

$x(t+1)=I x(t)+H v(t)+M u(t)+r(t)-p(t), \quad x(0)=x_{0}$

where the $n_{k}$-sized vectors $\boldsymbol{r}$ and $\boldsymbol{p}$ denote respectively the input flow of raw objects and the output flow of finished-products and $I$ the identity matrix. Each state component $x(i, k ; t)$ is integer (positive or negative) and each input component is a non negative integer. 


\section{Example}

Next the manufacturing matrix $M$ and the transport matrix $H$ are detailed. On the left side the bi-dimensional row indices $(i, k)$ are listed, $i$ being the storage unit index and $k$ the object type. On the upper side, the bi-dimensional column indices $(r, s)$ and $(h, l)$ are listed, $r$ being the manufacturing unit index, $s$ the manufacturing operation index, $h$ the transport unit index and $l$ the transport operation index.

$(r, s)$

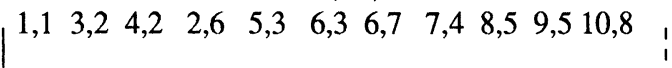

$\begin{array}{rrrrrrrrrrr}-1 & 0 & 0 & 0 & 0 & 0 & 0 & 0 & 0 & 0 & 0 \\ 0 & -1 & -1 & -1 & 0 & 0 & 0 & 0 & 0 & 0 & 0 \\ 0 & 0 & 0 & 0 & 0 & 0 & -2 & 0 & 0 & 0 & 0 \\ 0 & -2 & -2 & 0 & 0 & 0 & 0 & 0 & 0 & 0 & 0 \\ 1 & 0 & 0 & 0 & -1 & -1 & 0 & 0 & 0 & 0 & 0 \\ 1 & 0 & 0 & 0 & 0 & 0 & 0 & -2 & 0 & 0 & 0 \\ 0 & 1 & 1 & 0 & 0 & 0 & -1 & -2 & 0 & 0 & 0 \\ 0 & 0 & 0 & 1 & 0 & 0 & -1 & 0 & 0 & 0 & 0 \\ 0 & 0 & 0 & 0 & 1 & 1 & 0 & 0 & -1 & -1 & 0 \\ 0 & 0 & 0 & 0 & 0 & 0 & 2 & 1 & -1 & -1 & -1 \\ 0 & 0 & 0 & 0 & 0 & 0 & 0 & 0 & 1 & 1 & 0 \\ 0 & 0 & 0 & 0 & 0 & 0 & 2 & 0 & 0 & 0 & -1 \\ 0 & 0 & 0 & 0 & 0 & 0 & 0 & -1 & 0 & 0 & 0 \\ 0 & 0 & 0 & 0 & 0 & 0 & 0 & 0 & 0 & 0 & 1 \\ -1 & 0 & 0 & 0 & 0 & 0 & 0 & 0 & 0 & 0 & 0 \\ 0 & 0 & 0 & 0 & 0 & 0 & 0 & 0 & 1 & 1 & 0\end{array}$

$M$ $(h, l)$

\section{State controllability}

Since the state matrix is the identity matrix, the state controllability only depends on the input matrices. To this end a compact control matrix $B=[M H]$ is introduced, together with a single control vector $\boldsymbol{u}$ including manufacturing and transport commands. Then the state equation is rewritten as:

$$
x(t+1)=I x(t)+B u(t)+r(t)-p(t), \quad x(0)=x_{0}
$$

Definition. A state component $x(i, k ; t)$ is completely controllable if the row $(i, k)$ of $B$ contains at least a pair of components $b\left(i, k, r_{-}, s_{-}\right)$and $b\left(i, k ; r_{+}, s_{+}\right)$, with the property: $b\left(i, k, r_{-}, s_{-}\right)<0$, $b\left(i, k ; r_{+}, s_{+}\right)>0$.

According to this definition, there must exist at least two command components, one supplying and the other drawing objects, assuring that the state can reach any admissible value. The vector of the completely controllable components will be denoted by $x_{C}$.

Definition. A state component $x(i, k ; t)$ is partially (input/output) controllable if the $(i, k)$ th row of $B$ contains only negative or positive components. 
According to this definition, some objects can only be supplied or drawn upon factory commands; this is the case of finished-products (output factory objects) and raw materials (input factory objects). State components which are either completely or partially controllable are simply referred to as controllable. The vectors of partially controllable components will be denoted by $x_{I}$ (input) and $x_{O}$ (output); such components become completely controllable if the input vectors $\boldsymbol{r}$ and $\boldsymbol{p}$ are under control.

Definition. A state component $x(i, k ; t)$ is uncontrollable if the $(i, k)$ th row of $B$ is null. The vectors of uncontrollable components will be denoted by $\boldsymbol{x}_{N}$.

It is now possible to introduce the following state decomposition. The state equation (8) can be decomposed in the following form:

$$
\left|\begin{array}{l}
x_{C}(t+1) \\
x_{I}(t+1) \\
x_{O}(t+1) \\
x_{N}(t+1)
\end{array}\right|=\left|\begin{array}{llll}
I & 0 & 0 & 0 \\
0 & I & 0 & 0 \\
0 & 0 & I & 0 \\
0 & 0 & 0 & I
\end{array}\right|\left|\begin{array}{c}
x_{C}(t) \\
x_{f}(t) \\
x_{O}(t) \\
x_{N}(t)
\end{array}\right|+\left|\begin{array}{c}
B_{C} \\
B_{I} \\
B_{O} \\
0
\end{array}\right| u(t)+r(t)-p(t)
$$

where $B_{P} \geq 0$ and $B_{O} \leq 0$.

Definition. The state equation (8) is reduced to minimal form if and only if all the state components are either completely or partially controllable.

For instance, the matrices $M$ and $H$ in equation (7) correspond to a state vector in minimal form. The previous controllability definitions can be applied separately to transport and manufacturing controls.

\subsection{The steady state solution}

\section{The steady state equation}

Under steady conditions, the input vectors (i.e. the commands dispatched by the control system) and the state vector of the storage units are constant. That means:

$u(t)=\underline{u}, v(t)=\underline{v}, x(t)=\underline{x}, \quad r(t)=\underline{r}, p(t)=\underline{p}$

Applying such equalities to state equation (8), the following steady state condition is obtained:

$$
H \underline{v}+M \underline{u}+\underline{\boldsymbol{r}}-\underline{p}=0
$$

setting up a constraint between transport and manufacturing commands, raw objects supply and production yield during a generic time step.

In Section 3 the concept of manufacturing plan of a product map has been introduced. Manufacturing plans are independent of factory network, being only related to objects and manufacturing operations. In practice they assume a factory network to have only one random-access store and at least one manufacturing unit for each operation. Due to single- 
storage assumption a manufacturing plan does not need transport units. An assignment problem translates the manufacturing plan into a production plan by assigning objects to actual storage units and manufacturing operations to actual manufacturing units; at this stage provision is made also of transport operations and units. If the assignment is not unique as in the above example, i.e. the same operation can be assigned to more manufacturing units, the problem includes some degrees of freedom to be solved. The following computation steps are necessary to produce a steady state production plan $\{\underline{\boldsymbol{r}}, \boldsymbol{p}, \underline{\boldsymbol{u}}, \underline{\boldsymbol{v}}\}$, given the quintuple of the relevant manufacturing plan $\{W(\boldsymbol{A}), \boldsymbol{u}(W), \boldsymbol{y}(W), \boldsymbol{n}(W), \tau(w W\}$,

- Given the product mix $y(W)$ and the Bill of Materials, the object assignment to storage units determines the vectors $\underline{r}$ and $\boldsymbol{p}$.

- Given the repetition vector $\boldsymbol{m}(W)$, the operation assignment to manufacturing units determines the vector $\underline{\boldsymbol{u}}$ and the matrix $W$, after solution of the degrees of freedom.

- If the vector $W \underline{\boldsymbol{u}}+\underline{\boldsymbol{r}}-\boldsymbol{p}=\underline{\boldsymbol{h}}$ is null, no transport operation and consequently no transport unit is needed. Otherwise they must be defined, resulting in the matrix $H$. Finally the transport vector $\underline{\boldsymbol{v}}$ is solved; $\underline{\boldsymbol{v}}$ is called the steady-state transport plan.

\section{Example}

Coming back to the previous example, assume the mix variables introduced in Section 3 to have the following values: $x=1$ and $y=1$. The corresponding steady-state solution is reported in the following equation, by listing the values of the steady-state vectors $\underline{\boldsymbol{r}} \boldsymbol{\underline { p }}$ and $\underline{\boldsymbol{h}}$. In the same equation the manufacturing control vector $\underline{u}$ resulting from the assignment of the plan repetition $\boldsymbol{m}(W)$ is reported. The assignment criteria are not mentioned here, but they mainly correspond to force balance of manufacturing times. To this end the manufacturing time vector $\underline{\tau}$ required by each pair (unit, operation) and computed from the manufacturing times defined in Section 3 is reported; the total manufacturing time of each pair (unit, operation) has been computed by assuming series composition on each unit.

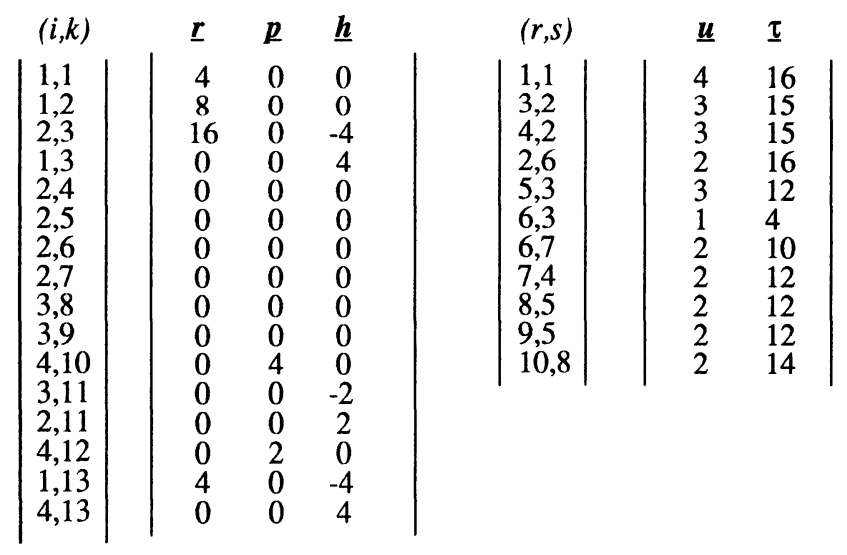

Different considerations apply to the above solution. 
- A transport plan $\underline{\boldsymbol{v}}$ is needed since $\underline{\boldsymbol{h}} \neq \mathbf{0}$. Indeed three different objects, namely $k=3,11,13$, need to be transported between storage units with a quantity listed in the same vector $\underline{\boldsymbol{h}}$. The transport units together with the transport matrix $H$ satisfy the requirements of $\underline{h}$. The transport plan is obtained after having defined the input and output vectors of each transport operation: in this case the following transport plan holds: $\underline{v}=\left[\begin{array}{lll}2 & 1 & 2\end{array}\right]^{T}$.

- The manufacturing times of the vector $\underline{\tau}$ can be used to select the sampling step of the model; the manufacturing time of the production plan is obtained by assuming that operations on different manufacturing units are composed in parallel and operations on the same one are composed in series. Applying algebra notations to the pair $(r, s)=($ unit,operation) the following aggregate operation, called production cycle, $\underline{W}$ result:

$$
\underline{W}=(1,1)^{4}+(3,2)^{3}+(4,2)^{3}+(2,6)^{2}+(5,3)^{3}+(6,3)(6,7)^{2}+(7,4)^{2}+(8,5)^{2}+(9,5)^{2}+(10,8)^{2}
$$

It is easy to verify that the manufacturing time holds $\tau(\underline{W})=16$. If also transport operations can be performed in parallel, such a time interval can be used to fix the sampling step $T$, i.e. $T \geq \tau(\underline{W})$.

\subsection{Dynamic properties}

\section{Open-loop control.}

Recall the factory state equation (8) and assume it has been reduced to minimal form:

$$
x(t+1)=I x(t)+B u(t)+r(t)-p(t), \quad x\left(t_{0}\right)=x_{0}
$$

First, assume that the input vectors $\boldsymbol{u}(t), \boldsymbol{r}(t)$ and $\boldsymbol{p}(t)$ are planned independently of the current state; e.g. they are the result of an optimized off-line plan. As long as there is no conflict with the physical constraints on the state vector $x(t), 0 \leq x(t) \leq \bar{x}$ or, more generally, as long as the commands are kept within their feasibility range, the above state equations remain linear and all their eigenvalues are unitary or equal to the complex value $(1,0)$. In this case, the state equation (14) correspond to a bank of $N$ adders, one for each state component; they are interconnected through the input vectors, but not interacting at the state level. Hence the following simple result can be stated.

Result. If the input vectors $\boldsymbol{u}(t), \boldsymbol{r}(t)$ and $\boldsymbol{p}(t)$ in equation (14) are state-independent (openloop control), the same equation is bounded-input unstable, i.e. at least one bounded input exists producing an unbounded state vector. This means that the state variables, i.e. the stock levels, are subject to uncontrolled drifts.

Note that such a situation, corresponding to an open-loop production control, is unrealistic, since any real factory neither operates nor could operate in this way.

\section{Perfect closed-loop control}

If instead the command vectors $\boldsymbol{u}(t), \boldsymbol{r}(t)$ and $\boldsymbol{p}(t)$ are state-dependent, i.e. the production control is closed-loop, then the eigenvalues of the state equations are moved away from the open-loop values and the state dynamics is modified. In this case, the state equation (14), 
modified as follows, represent an autonomous dynamic system:

$x(t+1)=x(t)+B u[x(t)]+r[x(t)]-p[x(t)], x\left(t_{0}\right)=x_{0}$

The resulting dynamics highly depends on the type of feedback control strategy, whose complete study is outside the scope of the present paper. Only very preliminary results will be presented without formal proof.

Definition. A feedback control strategy $u[x(t)], r[x(t)], p[x(t)]$ is perfect if it shifts the eigenvalues of equation (15) to the origin $(0,0)$.

In general perfect control strategies are not unique. Under perfect control, no matter which is the initial state value, are capable of reaching their steady-state condition in a finite number $N$ of sampling steps. It can also be proved that the transient interval $N$ is the same interval necessary to produce the earliest finished-product, by starting production with zero initial state (zero stock level).

\section{Push and FIFO control strategy}

The following simple closed-loop control strategies are now assumed:

- The transport command vector $v(t)$ is computed according to a push strategy, i.e. the semifinished products, as soon as they are available, are moved and queued into the input storage unit of the manufacturing units, where they have to undergo the subsequent manufacturing operations detailed in the product map.

- The manufacturing command vector $\boldsymbol{u}(t)$ is computed according to a FIFO strategy, i.e. the next operation of any manufacturing unit is decided by the first objects of the input queues, which are managed with a first-in-first-out rule.

\section{Fixture-free product map.}

Under such strategies, if the product map does not envisage re-circulation of objects, that is to say, if fixtures or objects to be re-utilized do not exist, the state equations take a tree-like structure, free of interactions capable of generating complex dynamic effects. The output stores of any manufacturing unit are immediately emptied as soon as the manufacturing units have fed them. They are therefore characterized by a zero eigenvalue. The input stores instead, if their current state value is positive, are emptied at a constant rate, which is independent of the state value or, in other words, independent of the queue of the waiting operations. Hence, their eigenvalues remain blocked at the open-loop value $(1,0)$, with a clear tendency toward process instability.

This is a well-known fact. If one keeps on feeding the production process at the rate of the first production stage, that is at the rate of the operating units supplied without limitations by the raw object quantity $\boldsymbol{r}$, the production will end up into instability (intermediate stores exploding) in all cases in which the bottleneck will not exactly coincide with the first stage manufacturing units.

In order to avoid instability, the supply of raw materials should be made dependent upon the state of the input stores of the bottleneck workstations. In this case the design of the closed-loop control shall coincide with a delay-line control, but the design will be not at all 
trivial if a single, well defined bottleneck does not exist.

\section{Re-circulating product map.}

Whenever fixtures exist, such to have a closed-loop re-circulation, all stores in the loop are automatically stabilized. The loop of stores having re-circulating objects cannot however be modelled by linear state equations, because the feedback control strategy is inherently non linear. Some preliminary results can already be stated:

- The dynamic properties of such a production process depend on the number of the available fixtures.

- The upper stock level of the stores distributed along the production process can not go beyond the total number of available fixtures.

- The production rate decreases as far as the number of the available fixtures is reduced. When the total number of fixtures becomes large enough, the production rate reaches the bottleneck rate.

\section{CONCLUSIONS}

The model presented in this paper appears a simple and at the same time powerful tool for the analysis of the dynamic properties of manufacturing plants and of their real-time production control systems.

\section{REFERENCES}

Canuto, E., Donati F. and Vallauri M. (1993) Factory modelling and production control. International Journal of Modelling and Simulation, 13, 162-166.

Canuto E., Donati F. and Vallauri M. (1994) A new approach to modelling manufacturing systems, in Proc. 27th ISATA Conf. on Lean Agile Manufacturing in the Automotive Industries, Aachen (Germany), 317-324.

\section{BIOGRAPHY}

The author is currently associate professor at Politecnico di Torino, where he teaches Automatic Control and Industrial Automation.

His basic research activity is concerned with the automatic control of uncertain and complex systems. He also applies theory and methods of automatic control in different fields: discrete manufacturing, urban traffic, robots and measuring machines calibration, spacecraft attitude estimation and control, industrial process control, economics.

His present research activity in the discrete manufacturing field is partly done within the ESPRIT Basic Research Project HIMAC 8141, supported by the European Community and coordinated by the an Italian company, EICAS Automazione spa, Torino.

$\mathrm{He}$ is author of more than 70 publications. 